PROCEEDINGS OF THE

AMERICAN MATHEMATICAL SOCIETY

Volume 30, Number 2, October 1971

\title{
THE EQUIVALENCE OF THE LEAST UPPER BOUND PROPERTY AND THE HAHN-BANACH EXTENSION PROPERTY IN ORDERED LINEAR SPACES ${ }^{1}$
}

\author{
TING-ON TO
}

\begin{abstract}
Let $V$ be a partially ordered (real) linear space with the positive wedge $C$. It is known that $V$ has the least upper bound property if and only if $V$ has the Hahn-Banach extension property and $C$ is lineally closed. In recent papers, W. E. Bonnice and R. J. Silverman proved that the Hahn-Banach extension and the least upper bound properties are equivalent. We found that their proof is valid only for a restricted class of partially ordered linear spaces. In the present paper, we supply a proof for the general case. We prove that if $V$ contains a partially ordered linear subspace $W$ of dimension $\geqq 2$, whose induced wedge $K=W \cap C$ satisfies $K \cup(-K)$ $=W$ and $K \cap(-K)=\{$ zero vector $\}$, then $V$ fails to have the Hahn-Banach extension property. From this the desired result follows.
\end{abstract}

1. Introduction. In [1] W. E. Bonnice and R. J. Silverman proved a theorem which states that in a (partially) ordered (real) linear space the least upper bound property and the Hahn-Banach extension property are equivalent. We indicated in [3, p. 165] that their proof is only valid for a restricted class of ordered linear spaces. The purpose of this paper is to supply a proof for the general case.

2. Definitions and preliminary lemmas. In this paper, we consider linear spaces over the field $R$ of real numbers. A nonempty subset $C$ of a linear space $V$ is said to be a wedge if $u, v \in C$ and $t \in R, t \geqq 0$, imply that $u+v$ and $t u$ are in $C$. If $V$ is ordered by a vector ordering $\geqq$, then the set $C=\{v: v \geqq \theta$, the zero element of $V\}$ is a wedge and is called the positive wedge of $V$ determined by $\geqq$. Conversely, a wedge $C$ in $V$ determines a vector ordering $\geqq$ by taking $a \geqq b$ if and only if $a-b \in C$. Therefore a wedge $C$ uniquely determines and is determined by a vector ordering $\geqq$.

A wedge $C$ is said to be sharp if $u \in C$ and $-u \in C$ imply that

Received by the editors November 18, 1969.

AMS 1969 subject classifications. Primary 4620, 4650; Secondary 0620.

Key words and phrases. Least upper bound property, Hahn-Banach extension property, lineally closed wedges, semispace-wedges, lexicographically ordered linear spaces.

1 This paper is part of the author's Ph.D. thesis prepared at the University of Saskatchewan under the supervision of Professor P. M. Cuttle. The author wishes to express his thanks to Professor P. M. Cuttle for his guidance and encouragement.

Copyright (@) 1971, American Mathematical Society 
$u=\theta$. It follows that the vector ordering $\geqq$ determined by the wedge $C$ is antisymmetric (i.e., $a \geqq b \geqq a$ implies $a=b$ ) if and only if $C$ is sharp.

Following [1], [2] we use the notation $(V ; C)$ to denote a (partially) ordered linear space (OLS) $V$ with the positive wedge $C$. An OLS $(V ; C)$ is said to have the least upper bound property (LUBP) if every set of elements of $V$ with an upper bound has a least upper bound (not necessarily unique). An OLS $(V ; C)$ is said to have the Hahn-Banach extension property (HBEP) if given (1) a linear space $X,(2)$ a linear subspace $Y$ of $X,(3)$ a function $p: X \rightarrow V$ which is sublinear (i.e., subadditive and positively homogeneous), and (4) a linear function $f: Y \rightarrow V$ such that $p(y)-f(y) \in C$ for all $y \in Y$, then there is a linear extension $F: X \rightarrow V$ of $f$ such that $p(x)-F(x) \in C$ for all $x \in X$.

A wedge $C$ in a linear space $V$ is said to be lineally closed if the intersection of $C$ with every line in $V$ is a closed set in the natural topology of the line. A wedge $C$ in a linear space $V$ is said to be a semispace-wedge if $C \cup(-C)=V$ and $C \cap(-C)=\{\theta\}$. An OLS $(V$; $C$ ) is said to be a lexicographically ordered linear space (LOLS) if the linear space $V$ is of dimension $\geqq 2$ and if the positive wedge $C$ is a semispace-wedge. It is clear that any ordered linear subspace of dimension $\geqq 2$ of a LOLS is still a LOLS.

Some preliminary lemmas concerning the above concepts are stated as follows:

Lemma A [4, p. 9]. Let $(V ; C)$ be an OLS, $X$ a linear space, $Y$ a linear subspace of $X$ such that the codimension of $Y$ in $X$ is $1, p$ a sublinear function from $X$ into $V$, and let $f$ be a linear function from $Y$ into $V$ such that $p(y)-f(y) \in C$ for all $y \in Y$. Then $f$ has a linear extension $F$ from $X$ into $V$ such that $p(x)-F(x) \in C$ for all $x \in X$ if and only if for each $x_{0} \in X \sim Y$ there exists $v_{0} \in V$ such that $-p\left(-y-x_{0}\right)-f(y) \leqq v_{0}$ $\leqq p\left(y^{\prime}+x_{0}\right)-f\left(y^{\prime}\right)$ for every $y, y^{\prime} \in Y$.

Lemma B ([4, p. 105], [5]). An OLS $(V ; C)$ has the LUBP if and only if $(V ; C)$ has the HBEP and $C$ is lineally closed.

Therefore by Lemma B, the equivalence of the LUBP and the HBEP in an OLS $(V ; C)$ will be established if we can show that the HBEP implies that the wedge $C$ is lineally closed (we will do this in $\S 3$, Lemma 4). To this end, the following lemma will be used.

Lemma C [1, pp. 844-845]. If an OLS $(V ; C)$ has the HBEP and if the positive wedge $C$ is not lineally closed, then there exists a 2-dimensional lexicographically ordered linear subspace of $(V ; C)$. 
3. Main theorem. We begin with the following lemmas.

Lemma 1. Let $(V ; C)$ be an OLS. If there is a family $\left\{\left(V_{\xi} ; C_{\xi}\right)\right\}_{\xi \in E}$ of ordered linear subspaces of $(V ; C)$, where $E$ is a nonempty set of indices ordered by a linear order $\prec$, satisfying the following conditions:

(i) the induced wedges $C_{\xi}=V_{\xi} \cap C, \xi \in E$, are semispace-wedges;

(ii) for every $\xi, \eta \in E, C_{\xi}<\left(C_{\eta} \sim\{\theta\}\right)^{2}$ if and only if $\xi \prec \eta$;

(iii) $V=\sum_{\xi \in E} V_{\xi}$, the direct sum of $V_{\xi}, \xi \in E$;

then $C$ is a semispace-wedge in $V$.

Proof. It is clear that for each nonzero element $v \in V, v$ has a unique representation $v=\sum_{i=1}^{k} \lambda_{\xi_{i}} v_{\xi_{i}}$ for some $\lambda_{\xi_{i}}=1$ or $-1, v_{\xi_{i}} \in C_{\xi_{i}}$ $\sim\{\theta\}, \xi_{i} \in E, i=1,2, \cdots, k$, with $\xi_{1} \prec \xi_{2} \prec \cdots \prec \xi_{k}$.

Since $\left(C_{\xi_{k}} \sim\{\theta\}\right)>C_{\xi_{i}}, i=1,2, \cdots, k-1$,

$$
v=v_{\xi_{k}}-\sum_{i=1}^{k-1}\left(-\lambda_{\xi_{i}}\right) v_{\xi_{\imath}}>\theta, \quad \text { if } \lambda_{\xi_{k}}=1
$$

and

$$
v=\sum_{i=1}^{k-1} \lambda_{\xi_{i}} v_{\xi_{i}}-v_{\xi_{k}}<\theta, \quad \text { if } \lambda_{\xi_{k}}=-1 .
$$

This shows that $V=C \cup(-C)$ and $C \cap(-C)=\{\theta\}$, and hence $C$ is a semispace-wedge in $V$.

The following two corollaries follow immediately from the lemma.

Corollary 1.1. Let $(V ; C)$ be an OLS such that $C$ is sharp. If there is a Hamel basis $B=\left\{b_{\xi}\right\}_{\xi \in E}$ of $V$, where $E$ is a nonempty set of indices ordered by a linear order $\succ$, such that $B>\theta$ and such that $\alpha b_{\eta}>\beta b_{\xi}$ for all real numbers $\alpha>0, \beta>0$, and for every $\eta, \xi \in E$ with $\eta \succ \xi$, then $C$ is $a$ semispace-wedge in $V$.

Corollary 1.2. Let $(V ; C)$ be an n-dimensional OLS such that $C$ is sharp. If there is a basis $\left\{b_{i}\right\}_{i=1}^{n}$ of $V$ such that $\alpha_{1} b_{1}>\alpha_{2} b_{2}>\cdots>\alpha_{n} b_{n}$ $>\theta$ for all $\alpha_{i}>0, i=1,2, \cdots, n$, then $C$ is a semispace-wedge. Moreover, $C$ can be expressed as the set

$$
\left\{\sum_{i=1}^{n} \lambda_{i} b_{i}: \lambda_{i} \in R, i=1,2, \cdots, n, \text { and the first } \lambda_{i} \text { not to vanish is positive }\right\} .
$$

The above corollary and its converse are well-known results [6], [7].

' Throughout $A \leqq B$ signifies that $a \leqq b$ for all $a \in A$ and $b \in B$; and $c \leqq A$ signifies that $c \leqq a$ for all $a \in A$. 
LEMMA 2. If $(V ; C)$ is a LOLS, $\left\{v_{i}\right\}_{i=1}^{n}$ is a basis of an $n$-dimensional $(n \geqq 1)$ linear subspace $V_{n}$ of $V$ such that $\theta<\alpha_{1} v_{1}<\alpha_{2} v_{2}<\cdots<\alpha_{n} v_{n}$ for all $\alpha_{i}>0, i=1,2, \cdots, n$, and $V_{n+m}$ is an $(n+m)$-dimensional $(m \geqq 1)$ linear subspace of $V$ containing $V_{n}$, then there exists a basis $\left\{v_{1}^{\prime}, v_{2}^{\prime}, \cdots, v_{n+m}^{\prime}\right\}$ of $V_{n+m}$ containing $\left\{v_{i}\right\}_{i=1}^{n}$ such that $\theta<\alpha_{1} v_{1}^{\prime}$ $<\alpha_{2} v_{2}^{\prime}<\cdots<\alpha_{n+m} v_{n+m}^{\prime}$ for all $\alpha_{i}>0, i=1,2, \cdots, n+m$.

The proof of Lemma 2, which is easily established by induction, is omitted.

Let $(V ; C)$ be a LOLS. The wedge $C$ is said to be a type (I) semispace-wedge if there exist $u_{1}, u \in C \sim\{\theta\}$ such that $u_{1}<\alpha u$ for every $\alpha>0$, and satisfying the following condition (I):

(I) There is no $v \in V$ such that $\alpha_{1} u_{1}<v<\alpha u$ for every $\alpha>0, \alpha_{1}>0$.

$C$ is said to be a type (II) semispace-wedge if it is not of type (I).

It is worth remarking that for the finite dimensional case, the positive wedge of any LOLS is a type (I) semispace-wedge but that for the infinite dimensional case, there exist many LOLS's each having its positive wedge which is a type (II) semispace-wedge.

The following lemma is the main result of this paper:

Lemma 3. Let $(V ; C)$ be an OLS. If $(V ; C)$ contains a lexicographically ordered linear subspace $(W ; K)$, where $K=W \cap C$, then $(V ; C)$ fails to have the HBEP. 's

Proof. We first assume that the positive wedge $C$ is sharp.

A Zorn's Lemma argument guarantees the existence of a maximal lexicographically ordered linear subspace $\left(W^{*} ; K^{*}\right)$ of $(V ; C)$ containing $(W ; K)$ where $K^{*}=W^{*} \cap C$. We shall show that if $(V ; C)$ has the HBEP, then $\left(W^{*} ; K^{*}\right)$ fails to be maximal. This contradiction will establish the lemma in case $C$ is sharp. To this end, we assume that $(V ; C)$ has the HBEP and consider the following two cases:

Case $1 . K^{*}$ is a type (I) semispace-wedge.

Let $u_{1}, u \in K^{*} \sim\{\theta\}$, such that $u_{1}<\alpha u$ for every $\alpha>0$, and satisfying the condition (I), i.e., there is no $w \in W^{*}$ such that $\alpha_{1} u_{1}<w<\alpha u$ for every $\alpha_{1}>0, \alpha>0$.

Let

$$
X=\left\{\left(t_{1}, t_{2}\right): t_{i} \in R, i=1,2\right\}=R_{2}, \quad Y=\left\{\left(0, t_{2}\right): t_{2} \in R\right\} .
$$

Define $P: X \rightarrow V$ by

$$
\begin{aligned}
& p\left(\left(t_{1}, t_{2}\right)\right)=-\left(t_{1}^{+} t_{2}^{+}\right)^{1 / 2} u_{1}+\left(\left|t_{2}\right|+t_{1}^{+}\right) u, \quad \text { if } t_{1}=t_{2} ; \\
& p\left(\left(t_{1}, t_{2}\right)\right)=-\left(t_{1}^{+} t_{2}^{+}\right)^{1 / 2} u_{1}+\left\{\left|t_{2}\right|+t_{1}^{+}-\left[\left(t_{2}^{+} \overline{t_{1}}\right) /\left(t_{2}-t_{1}\right)\right]\right\} u, \\
& \text { for all } t_{1} \neq t_{2},
\end{aligned}
$$


where $t^{+}=\sup \{t, 0\}$ and $t^{-}=\sup \{-t, 0\}$.

Define $f: Y \rightarrow V$ by $f\left(\left(0, t_{2}\right)\right)=t_{2} u$ for every $t_{2} \in R$.

Then $f$ is a linear function and $p(y)-f(y) \in K^{*}$ for every $y \in Y$ Moreover, referring to [2, p. 221, Case (2v) and p. 217, Example 2], $p$ is a sublinear function from $X$ into $W^{*}$. Note that

$$
\begin{aligned}
-p\left(\left(-1,-t_{2}\right)\right)-f\left(\left(0, t_{2}\right)\right) & =-2 t_{2} u, & & \text { if } t_{2} \geqq 0 ; \\
& =\left(-t_{2} /\left(1-t_{2}\right)\right) u, & & \text { if } t_{2}<0 ;
\end{aligned}
$$

and

$$
\begin{aligned}
p\left(\left(1, t_{2}^{\prime}\right)\right)-f\left(\left(0, t_{2}^{\prime}\right)\right) & =-\left(t_{2}^{\prime}\right)^{1 / 2} u_{1}+u, & & \text { if } t_{2}^{\prime} \geqq 0 ; \\
& =\left(-2 t_{2}^{\prime}+1\right) u, & & \text { if } t_{2}^{\prime}<0 .
\end{aligned}
$$

Let

$$
\begin{aligned}
& S=\left\{w \in W^{*}: w=\left(-t_{2} /\left(1-t_{2}\right)\right) u, t_{2} \leqq 0\right\}, \\
& T=\left\{w \in W^{*}: w=-\left(t_{2}^{\prime}\right)^{1 / 2} u_{1}+u, t_{2}^{\prime} \geqq 0\right\} .
\end{aligned}
$$

Then $S \leqq T$. Since $(V ; C)$ has the HBEP, by Lemma A there exists $v_{0} \in V$ such that $S \leqq v_{0} \leqq T$. We claim that this element $v_{0} \notin W^{*}$. Indeed, if there is $v_{0} \in W^{*}$ such that $S \leqq v_{0} \leqq T$, then $u-v_{0} \in W^{*}$ and $\left(t_{2}^{\prime}\right)^{1 / 2} u_{1} \leqq u-v_{0} \leqq\left(1 /\left(1-t_{2}\right)\right) u$ for all $t_{2}^{\prime}>0$ and $t_{2}<0$, a contradiction.

Let $v_{0}^{\prime}=u-v_{0}$, then $\alpha_{1} u_{1}<v_{0}^{\prime}<\alpha u$ for every $\alpha_{1}>0, \alpha>0$, and $v_{0}^{\prime} \notin W^{*}$. Let $V^{\prime}$ be the subspace of $V$ spanned by $W^{*}$ and $v_{0}^{\prime}$.

We shall prove that the ordered linear subspace $\left(V^{\prime} ; C^{\prime}\right)$, where $C^{\prime}=V^{\prime} \cap C$, is a LOLS. Let $W_{2}=\operatorname{lin}\left\{u_{1}, u\right\},{ }^{3} V_{3}^{\prime}=\operatorname{lin}\left\{u_{1}, v_{0}^{\prime}, u\right\}$. Then, by Corollary 1.2, $\left(W_{2} ; K^{*} \cap W_{2}\right)$ and $\left(V_{3}^{\prime} ; C \cap V_{3}^{\prime}\right)$ are LOLS's.

Consider each nonzero element $w^{*}+\lambda v_{0}^{\prime} \in V^{\prime}$, where $w^{*} \in W^{*}$, $\lambda \in R$. If $w^{*} \in W_{2}$, then $w^{*}+\lambda v_{0}^{\prime} \in V_{3}^{\prime}$ and hence $w^{*}+\lambda v_{0}^{\prime}$ belongs to one and only one of the wedges $C^{\prime} \cap V_{3}^{\prime}$ and $-C^{\prime} \cap V_{3}^{\prime}$. Thus, $w^{*}$ $+\lambda v_{0}^{\prime}$ belongs to one and only one of the wedges $C^{\prime}$ and $-C^{\prime}$. If $w^{*} \notin W_{2}$, let $W_{3}=\operatorname{lin}\left\{u_{1}, u, w^{*}\right\}$ and let $V_{4}^{\prime}=\operatorname{lin}\left(W_{3} \cup\left\{v_{0}^{\prime}\right\}\right)$. Since $\left(W_{3} ; W_{3} \cap K^{*}\right)$ is an ordered linear subspace of a LOLS $\left(W^{*} ; K^{*}\right)$, by Lemma 2, there is $w_{0}^{*} \in W_{3}$ such that $\left\{u_{1}, u, w_{0}^{*}\right\}$ forms a basis for $W_{3}$ and is such that either $\theta<\alpha w_{0}^{*}<u_{1}<\beta u$, or $\alpha u_{1}<u<\beta w_{0}^{*}$, or $\alpha u_{1}<w_{0}^{*}$ $<\beta u$, for every $\alpha>0, \beta>0$. By our hypothesis, the last case is excluded. In the first case, we have $\theta<\alpha w_{0}^{*}<u_{1}<\beta v_{0}^{\prime}<u$ for every $\alpha>0$, $\beta>0$; in the second case, we have $\alpha u_{1}<v_{0}^{\prime}<\beta u<w_{0}^{*}$ for every $\alpha>0$, $\beta>0$. Thus, by Corollary 1.2 , in both cases the ordered linear subspace $\left(V_{4}^{\prime} ; V_{4}^{\prime} \cap C^{\prime}\right)$ is a LOLS and hence $w^{*}+\lambda v_{0}^{\prime}$ belongs to one and only one of the wedges $V_{4}^{\prime} \cap C^{\prime}$ and $-\left(V_{4}^{\prime} \cap C^{\prime}\right)$. Thus $w^{*}+\lambda v_{0}^{\prime}$ belongs

Throughout lin $A$ signifies the linear hull of the set $A$. 
to one and only one of the wedges $C^{\prime}$ and $-C^{\prime}$. This shows that $V^{\prime}$ $=C^{\prime} \cup\left(-C^{\prime}\right)$ and $C^{\prime} \cap\left(-C^{\prime}\right)=\{\theta\}$. Thus, $\left(V^{\prime} ; C^{\prime}\right)$ is a LOLS with a semispace-wedge $C^{\prime}$ and hence $\left(W^{*} ; K^{*}\right)$ is not a maximal lexicographically ordered linear subspace in $(V ; C)$.

Case 2. $K^{*}$ is a type (II) semispace-wedge.

Let $u_{1}, u \in K^{*} \sim\{\theta\}$ be such that $u_{1}<\alpha u$ for every $\alpha>0$ and let $U$ $=\left\{w \in W^{*}: \beta u_{1}<w<\alpha u\right.$, for every $\left.\alpha>0, \beta>0\right\}$. Since $K^{*}$ is of type (II), $U$ is nonempty and has no maximal element relative to the order of $\left(W^{*} ; K^{*}\right)$. Moreover, by the maximal principle $U$ contains a maximal linearly independent subset $B$. Let $Y$ be the linear space spanned by $B$ and $u_{1}$, and let $X$ be the linear space spanned by $Y$ and $u$. Then $\left\{u_{1}\right\} \cup B$ and $\left\{u_{1}\right\} \cup B \cup\{u\}$ are Hamel bases of $Y$ and $X$, respectively. Thus, for each $x \in X$, there exists a finite subset $\left\{u_{\xi_{i}}\right\}_{i=1}^{k}$ of $B$ (where $k$ is some integer and $u_{\xi_{1}}<u_{\xi_{2}}<\cdots<u_{\xi_{k}}$ relative to the ordering of $(V ; C))$, so that $x$ has the unique representation, $x=t_{1} u_{1}$ $+\sum_{i=1}^{k} t_{\xi_{1}} u_{\xi_{i}}+t u$, where $t_{1}, t$ are real numbers and $t_{\xi_{i}}, i=1,2, \cdots, k$, are nonzero real numbers if $x \notin \operatorname{lin}\left\{u_{1}, u\right\}$; they are all zero if $x$ $\in \operatorname{lin}\left\{u_{1}, u\right\}$.

Let $f: Y \rightarrow V$ be a linear function defined by

$$
f(y)=t_{1} u_{1}+\sum_{i=1}^{k} t_{\xi_{i}} u_{\xi_{i}}, \quad \text { for all } y=t_{1} u_{1}+\sum_{i=1}^{k} t_{\xi_{i}} u_{\xi_{i}} \in Y,
$$

and let $p: X \rightarrow V$ be a function defined by

$$
p(x)=p_{1}(x)+p_{2}(x)+p_{3}(x) u, \text { for every } x=t_{1} u_{1}+\sum_{i=1}^{k} t_{\xi_{i}} u_{\xi_{i}}+t u \in X,
$$

where

$$
\begin{aligned}
& p_{1}(x)=t_{1}^{+} u_{1}+\sum_{i=1}^{k-1} t_{\xi_{i}}^{+} u_{\xi_{i}} ; \\
& p_{2}(x)=\left(t_{\xi_{k}}^{+}-t^{-} t_{\xi_{k}}^{+} /\left(-t+t_{\xi_{k}}\right)\right) u_{\xi_{k}} \quad \text { if } t \neq t_{\xi_{k}} ; \\
& p_{2}(x)=t_{\xi_{k}}^{+} u_{\xi_{k}} \quad \text { if } t=t_{\xi_{k}} ; \\
& p_{3}(x)=p_{3}^{\prime}\left(\left(t_{1}, t\right)\right)=t_{1}^{+}+t^{+}-\bar{t}_{1} t^{+} /\left(t-t_{1}\right), \quad \text { if } t \neq t_{1},
\end{aligned}
$$

and

$$
p_{3}(x)=p_{3}^{\prime}\left(\left(t_{1}, t\right)\right)=t_{1}^{+}+t^{+} \quad \text { if } t=t_{1} ;
$$

where $t^{+}=\sup \{t, 0\}$ and $t^{-}=\sup \{-t, 0\}$. 
It follows that $p(y)-f(y) \in C$ for all $y \in Y$. We claim that $p$ is sublinear from $X$ into $V$. It is clear that $p_{1}(x), p_{2}(x)$ and $p_{3}(x)$ are positively homogeneous and that $p_{1}(x)$ is also subadditive. Since $u_{\xi_{k}}<\alpha u$ for every $\alpha>0, u_{\xi_{k}} \in B$, it remains to show that $p_{3}$ is subadditive and that $p_{2}(x)+p_{2}\left(x^{\prime}\right) \geqq p_{2}\left(x+x^{\prime}\right)$ whenever $p_{3}(x)+p_{3}\left(x^{\prime}\right)=p_{3}\left(x+x^{\prime}\right)$ for every $x, x^{\prime} \in X$. It requires detailed computations to show analy tically that $p_{3}$ is subadditive. However, the graph of $p_{3}^{\prime}$ makes this obvious. Hence the computations will be omitted.

Also, from the graph of $p_{3}^{\prime}$, it is clear that $p_{3}\left(x+x^{\prime}\right)=p_{3}(x)+p_{3}\left(x^{\prime}\right)$, where

$$
x=t_{1} u_{1}+\sum_{i=1}^{k} l_{\xi_{i}} u_{\xi_{i}}+t u, \quad x^{\prime}=t_{1}^{\prime} u_{1}+\sum_{i=1}^{k^{\prime}} t_{\eta_{i}}^{\prime} u_{\eta_{i}}+t^{\prime} u
$$

if and only if

(a) $t_{1}=r t_{1}^{\prime}$ and $t=r t^{\prime}, r>0$, or

(b) $t_{1} \geqq 0, t \geqq 0, t_{1}^{\prime} \geqq 0, t^{\prime} \geqq 0$, or

(c) $t_{1} \geqq 0, t \leqq 0, t_{1}^{\prime} \geqq 0, t^{\prime} \leqq 0$, or

(d) $t_{1} \leqq 0, t \leqq 0, t_{1}^{\prime} \leqq 0, t^{\prime} \leqq 0$.

Simple direct computations show that in these cases $p_{2}\left(x+x^{\prime}\right)$ $\leqq p_{2}(x)+p_{2}\left(x^{\prime}\right)$. Thus, $p$ is sublinear from $X$ into $V$. Furthermore,

$-p\left(r u_{\xi_{k}}-u\right)-f\left(-r u_{\xi_{k}}\right)=(r /(1+r)) u_{\xi_{k}}, \quad$ for every $u_{\xi_{k}} \in B, r>0$;

and

$$
p\left(-s u_{1}+u\right)-f\left(-s u_{1}\right)=(1 /(1+s)) u+s u_{1}, \quad \text { for all } s \geqq 0 .
$$

Let $M=\left\{(r /(1+r)) u_{\xi_{k}}: u_{\xi_{k}} \in B, \quad r>0\right\}$ and let $N=\{(1 /(1+s)) u$ $\left.+s u_{1}: s \geqq 0\right\}$. Then $M<N$. By Lemma A, since $(V ; C)$ has the HBEP, there is $v_{0} \in V$ such that $M \leqq v_{0} \leqq N$.

We claim that this element $v_{0} \notin W^{*}$. For suppose on the contrary that $v_{0} \in W^{*}$, then from $(r /(1+r)) u_{\xi_{k}} \leqq v_{0} \leqq(1 /(1+s)) u+s u_{1}$, for every $r>0, s>0$ and $u_{\xi_{k}} \in B$, we have (i) $u, v_{0} \in K^{*} \sim\{\theta\}$ with $v_{0}<\alpha u$ for all $\alpha>0$, and (ii) for every $u^{\prime} \in U$ there exists $\alpha^{\prime}>0$ such that $\alpha^{\prime} u^{\prime}<v_{0}$. Clearly, (i) and (ii) are contradictory since $K^{*}$ is a type (II) semispace-wedge.

In order to show that $\left(W^{*} ; K^{*}\right)$ is not maximal, let $V^{\prime}=$ $\operatorname{lin}\left(W^{*} \cup\left\{v_{0}\right\}\right)$. We claim that $\left(V^{\prime} ; C^{\prime}\right)$, where $C^{\prime}=V^{\prime} \cap C$, is a LOLS. Let $W^{\prime}=\operatorname{lin}\left(B \cup\left\{u_{1}\right\}\right), W^{\prime \prime}=\operatorname{lin}\left(B \cup\left\{u_{1}, u\right\}\right), V^{\prime \prime}=\operatorname{lin}\left(W^{\prime \prime} \cup\left\{v_{0}\right\}\right)$. Then $\left(W^{\prime} ; W^{\prime} \cap K^{*}\right)$ and $\left(W^{\prime \prime} ; W^{\prime \prime} \cap K^{*}\right)$ are lexicographically ordered linear subspaces of $\left(W^{*} ; K^{*}\right)$. Furthermore, the ordered linear subspace $\left(V^{\prime \prime} ; V^{\prime \prime} \cap C\right)$ of $(V ; C)$ is a LOLS. For, if $u_{\xi} \in B$ we can choose $u^{\prime} \in U$ such that $\lambda u_{\xi}<\alpha u^{\prime}<\beta u$ for all $\lambda>0, \alpha>0, \beta>0$. 
Since $u^{\prime} \in U$ and $\frac{1}{2} u_{\xi}<v_{0}$ for all $u_{\xi} \in B$, there exists $\alpha^{\prime}>0$ such that $\alpha^{\prime} u^{\prime}<v_{0}$. Also, $M \leqq v_{0} \leqq N$ implies that $v_{0}<\beta u$ for all $\beta>0$. Thus, $\lambda u_{\xi}<v_{0}<\beta u$ for all $\lambda>0, \beta>0$. It follows that $W^{\prime} \cap K^{*}<\alpha v_{0}<\beta u$ for all $\alpha>0, \beta>0$. Therefore, by Lemma $1,\left(V^{\prime \prime} ; V^{\prime \prime} \cap C\right)$ is a LOLS as was asserted.

To see that $C^{\prime}$ is a semispace-wedge in $V^{\prime}$, we consider each nonzero element $v=w^{*}+\lambda v_{0} \in V^{\prime}$, where $w^{*} \in W^{*}, \lambda \in R$. If $w^{*} \in W^{\prime \prime}$, then $v \in V^{\prime \prime}$ and hence $v$ belongs to one and only one of the wedges $V^{\prime \prime} \cap C^{\prime}$ and $-\left(V^{\prime \prime} \cap C^{\prime}\right)$. Thus, $v$ belongs to one and only one of the wedges $C^{\prime}$ and $-C^{\prime}$. If $w^{*} \notin W^{\prime \prime}$, let $W^{\prime \prime \prime}=\operatorname{lin}\left(W^{\prime \prime} \cup\left\{w^{*}\right\}\right), \quad V^{\prime \prime \prime}=$ $\operatorname{lin}\left(W^{\prime \prime} \cup\left\{v_{0}\right\}\right)$ and let $W_{3}^{*}=\operatorname{lin}\left\{u_{1}, u, w^{*}\right\}$.

Note that $\left(W_{3}^{*} ; W_{3}^{*} \cap K^{*}\right)$ is a lexicographically ordered linear subspace of $\left(W^{*} ; K^{*}\right)$; hence by Lemma 2 there is $w_{0}^{*} \in W_{3}^{*}$ such that $\left\{u_{1}, u, w_{0}^{*}\right\}$ forms a basis for $W_{3}^{*}$ and is such that either $\theta<\alpha w_{0}^{*}<u_{1}$ $<\beta u$, or $\alpha u_{1}<u<\beta w_{0}^{*}$, or $\alpha u_{1}<w_{0}^{*}<\beta u$, for every $\alpha>0, \beta>0$. Since $w^{*} \notin W^{\prime \prime}, w_{0}^{*} \notin W^{\prime \prime}$ and hence $w_{0}^{*} \notin \operatorname{lin} B$. Thus, the last case $\alpha u_{1}<w_{0}^{*}$ $<\beta u$, for every $\alpha>0, \beta>0$, is excluded. From the first case or the second case, we have $\theta<\alpha w_{0}^{*}<u_{1}<C_{B}<\beta v_{0}<u$ or $\alpha u_{1}<C_{B}<\beta v_{0}<u$ $<\gamma w_{0}^{*}$, for every $\alpha>0, \beta>0$ and $\gamma>0$, respectively, where $C_{B}=\lambda B$, $\lambda>0$. Therefore, by Lemma 1 , in both cases the induced wedge $C^{\prime \prime \prime}=V^{\prime \prime \prime} \cap C^{\prime}$ is a semispace-wedge in $V^{\prime \prime \prime}$ and hence $v=w^{*}+\lambda v_{0}$ belongs to one and only one of the wedges $C^{\prime \prime \prime}$ and $-C^{\prime \prime \prime}$. Thus, $v$ belongs to one and only one of the wedges $C^{\prime}$ and $-C^{\prime}$. This shows that $\left(V^{\prime} ; C^{\prime}\right)$ is a LOLS, and hence that $\left(W^{*} ; K^{*}\right)$ is not maximal. This completes the proof of the lemma in case $C$ is sharp.

In case $C$ is not sharp, let $V_{0}=\{v \in V: v \in C \cap-C\}$ and let $C_{0}$ $=V_{0} \cap C$. Since any lexicographical order is antisymmetric, $W \cap V_{0}$ $=\{\theta\}$. Therefore, there exists a subspace $V_{1}$ of $V$ containing $W$ which is complementary to $V_{0}$ in $V$. Let $C_{1}=V_{1} \cap C$. Then $C_{1}$ is sharp. Thus, by the result that we have just proved, $\left(V_{1}, C_{1}\right)$ fails to have the HBEP. Also, it is easy to verify that $(V ; C)$ has the HBEP if and only if $\left(V_{1}, C_{1}\right)$ has the HBEP. Therefore, $(V ; C)$ fails to have the HBEP.

LEMma 4. If an OLS $(V ; C)$ has the HBEP, then the positive wedge $C$ is lineally closed.

Proof. Assume that $(V ; C)$ has the HBEP and that the positive wedge $C$ is not lineally closed. Then, by Lemma $C$, there exists a 2 dimensional lexicographically ordered linear subspace of $(V ; C)$, and hence, by Lemma $3,(V ; C)$ fails to have the HBEP, a contradiction.

From Lemma B and Lemma 4, the following theorem is immediate: 
TheOREM. Let $(V ; C)$ be an OLS. Then $(V ; C)$ has the LUBP if and only if $(V ; C)$ has the $H B E P$.

\section{BIBLIOGRAPHY}

1. W. E. Bonnice and R. J. Silverman, The Hahn-Banach extension and the least upper bound properties are equivalent, Proc. Amer. Math. Soc. 18 (1967), 843-849. MR 35 \#5895.

2. - The Hahn-Banach theorem for finite dimensional spaces, Trans. Amer. Math. Soc. 121 (1966), 210-222. MR 32 \#2879.

3. T. O. To, $A$ note of correction to a theorem of W. E. Bonnice and R. J. Silverman, Trans. Amer. Math. Soc. 139 (1969), 163-166. MR 39 \#57.

4. M. M. Day, Normed linear spaces, 2nd rev. ed., Ergebnisse der Mathematik, Heft 21, Springer-Verlag, Berlin, 1958. MR $20 \# 1187$.

5. R. J. Silverman and Ti Yen, The Hahn-Banach theorem and the least upper bound property, Trans. Amer. Math. Soc. 90 (1959), 523-526. MR 21 \#1511.

6. P. C. Hammer, Maximal convex sets, Duke Math. J. 22 (1955), 103-106. MR $16,612$.

7. V. L. Klee, Jr., The structure of semispaces, Math. Scand. 4 (1956), 54-64. MR $18,330$.

University of Saskatchewan, Saskatoon, Saskatchewan, Canada

University of Canterbury, Christchurch, New Zealand 